\title{
US Children 'Learning Online' During COVID-19 Without the Internet or a Computer: Visualizing the Gradient by Race/Ethnicity and Parental Educational Attainment
}

Joseph Friedman, $\mathrm{MPH}^{1^{*}}$, Hunter York, $\mathrm{MPH}^{2}$, Ali Mokdad, PhD', Emmanuela Gakidou, PhD, MSc ${ }^{3}$

${ }^{1}$ Center for Social Medicine and Humanities, University of California, Los Angeles

${ }^{2}$ Department of Sociology, Princeton University

${ }^{3}$ Institute for Health Metrics and Evaluation, University of Washington

*Correspondence: Josephfriedman@mednet.ucla.edu

\begin{abstract}
The COVID-19 pandemic has caused unprecedented disruptions to education in the United States, with a large proportion of schooling moving to online formats, which has the potential to exacerbate existing racial/ethnic and socioeconomic disparities in learning. We visualize access to online learning technologies using data from the Household Pulse Survey from the early Fall 2020 schoolyear (August 19-October 26). We find that $10.1 \%$ of children participating in online-learning nationally did not have adequate access to the internet and a computer. Rates of inadequate access varied nearly twentyfold across the gradient of parental race/ethnicity and education, from $1.9 \%$ for children of Asian parents with a graduate degree, to $35.5 \%$ among children of Black parents with less than a high school education. These findings indicate alarming gaps in potential learning among US children. Renewed investments in equitable access to distance-learning resources will be necessary to prevent widening racial/ethnic and class learning disparities.
\end{abstract}

Keywords: Educational Inequality, Online learning, COVID-19, Racial/Ethnic Disparities 


\section{Article}

The COVID-19 pandemic has caused unprecedented disruptions to education globally and in the US, with a large proportion of schooling moving to distance-learning formats that require access to the internet and computer technology. We use data from the weekly Household Pulse Survey (US Census Bureau 2020), a rapidly deployed, representative sample of the US population, describing early trends in the Fall 2021 school period (August 19-October 26). We estimate that 58.1\% (57.5\% - 58.7\%) of US children participated in online learning. Of these, $10.1 \%(95 \% \mathrm{Cl}: 9.6 \%-10.6 \%)$ did not have adequate access to both the internet and a computer (Figure; see supplement for sample sizes and confidence intervals).

\section{The Gradient by Parental Race/Ethnicity and Education}

Lack of adequate access varied nearly twentyfold across the gradient of parental race/ethnicity and education, ranging from $1.9 \%(1.2 \%-2.7 \%)$ for children of Asian parents with a graduate degree, up to $35.5 \%$ (24.1\% - 47.0\%) among children of Black parents with less than high school education (Figure).

These rates varied fourfold by race/ethnicity, ranging from 3.8\% (2.8\% - 4.9\%) for Asian parents up to $15.6 \%(13.5 \%$ - $17.7 \%)$ for Black parents and fivefold by parental education, ranging from 3.9\% (3.5\% $4.3 \%)$ for parents with graduate degrees to $20.3 \%(16.5 \%-24.2 \%)$ for parents with less than high school education.

\section{Implications}

Even before COVID-19, the United States had profound and persistent disparities in educational attainment and learning by race and social class (York 2020). The results presented here concur with recent studies suggesting that that COVID-19 may be further exacerbating existing gaps in schooling (Bacher-Hicks, Goodman, and Mulhern 2020) and extends them to the Fall 2020 school period. Children expected to participate in online learning without adequate technology are highly unlikely to achieve significant learning compared to their peers for whom more resources are available. Falling behind in learning goals, they may also be more likely to leave school entirely.

By visualizing the gradient by parental race/ethnicity and education, we highlight that COVID-19 is likely potentiating the intergenerational propagation of gaps in educational outcomes, and moreover in a differential manner by race/ethnicity. These findings therefore demonstrate that disparities in remote learning during COVID-19 must be examined in an intersectional fashion (Bhopal and Preston 2012). Stratification by parental race and education revealed disparities along both dimensions, with specific subgroups exposed to large magnitude disparities. Further study should examine how racial/ethnic and social class differences also may vary by geography, potentially reflecting the diversity of educational approaches taken by municipal and state government in response to COVID-19-related disruptions.

As education is a key social determinant of health, and driver of economic opportunities, the implications of these widening education gaps are myriad, and may ripple out into disparities in numerous sectors of society (Lim et al. 2018). These trends highlight a need for renewed investments in ensuring universal access to distance-learning resources for all children in the United States, and may have implications for school districts and states making decisions regarding school closures. 


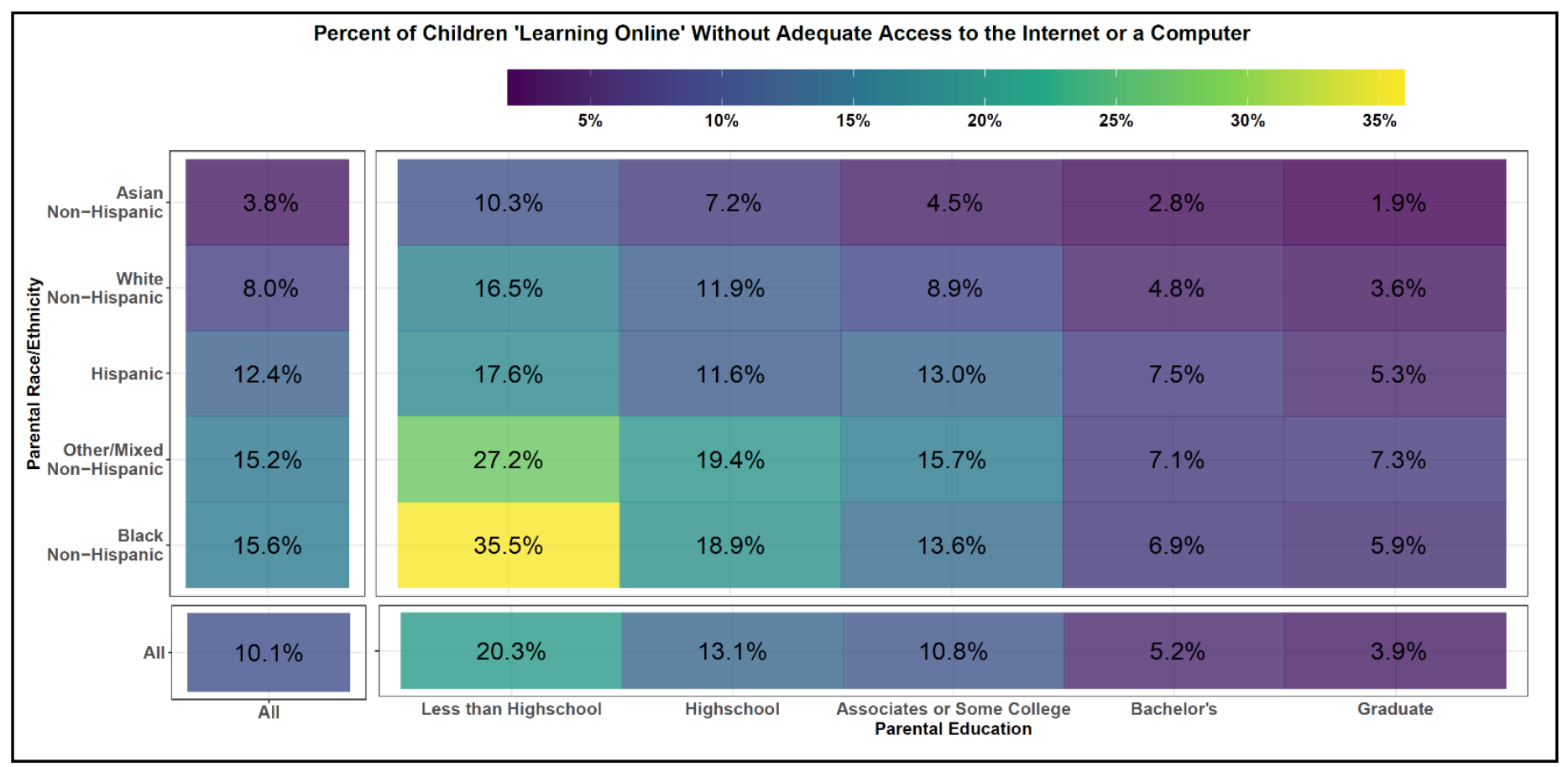

Figure. Percent of children (age 0-18) learning online whose parents reported they had inadequate access to the internet or a computer, in the Fall 2020 school period (August 19 - October 26), shown by parental race and parental education gradient. All intersections are shown as well as marginal values. All percentages represent survey-weighted values. See the supplement for confidence intervals, and more details regarding methodology.

\section{References}

Bacher-Hicks, Andrew, Joshua Goodman, and Christine Mulhern. 2020. Inequality in Household Adaptation to Schooling Shocks: Covid-Induced Online Learning Engagement in Real Time. w27555. National Bureau of Economic Research.

Bhopal, Kalwant, and John Preston. 2012. Intersectionality and "Race" in Education. Routledge.

Lim, Stephen S., Rachel L. Updike, Alexander S. Kaldjian, Ryan M. Barber, Krycia Cowling, Hunter York, Joseph Friedman, R. Xu, Joanna L. Whisnant, Heather J. Taylor, Andrew T. Leever, Yesenia Roman, Miranda F. Bryant, Joseph Dieleman, Emmanuela Gakidou, and Christopher J. L. Murray. 2018. "Measuring Human Capital: A Systematic Analysis of 195 Countries and Territories, 19902016." The Lancet. doi: 10.1016/S0140-6736(18)31941-X.

US Census Bureau. 2020. "Household Pulse Survey Public Use File (PUF)." Census.Gov. Retrieved November 22, 2020 (https://www.census.gov/programs-surveys/household-pulsesurvey/datasets.html).

York, Hunter W. 2020. "Race/Ethnicity and Geographic Disparities in Learning Adjusted Years of Schooling in the United States, 2009-2016." University of Washington. 


\section{Supplemental Materials for: "US Children 'Learning Online' During COVID-19 Without the Internet or a Computer: Visualizing the Gradient by Race and Parental Educational"}

\section{Steps Taken to Conduct the Analysis}

Public use microdata from the weekly Household Pulse Survey were obtained from the US Census Bureau: https://www.census.gov/programs-surveys/household-pulse-survey/datasets. html (accessed Nov 25, 2020).

1. Data from waves 13-17 were used, as they ask explicitly about the 2020-2021 school year.

2. Parental race and ethnicity were categorized into 5 groups using the RHISPANIC and RRACE variables. Race and ethnicity variables had no observed missingness.

3. Parental income was excluded as an analytical variable due to high missingness (22\%).

4. Parental education was included as an analytical variable given no apparent missingness, and high relevance to the study topic. It was categorized into 5 categories from the variable EEDUC.

5. A subset of the full dataset was used, consisting of only those households with at least 1 child currently enrolled in school, who answered affirmatively to the TEACH2 question: "Classes normally taught in person moved to a distance-learning format using online resources, either self-paced or in real time."

6. The outcome variable was defined using the variables COMPAVAIL and INTRNTAVAIL describing children's computer and internet access, respectively. Households reporting that both were "always available" or "usually available" were assigned a value of 0 , whereas for households in which either variable was reported as "sometimes available" "rarely available" and "never available" a value of 1 was assigned.

7. Missingness in either variable (COMPAVAIL and INTRNTAVAIL) was low, at $0.019 \%$ of the subset of households with children participating in online learning.

8. Percentages were calculated for all values shown in the figure. Survey weights were used for all values. The weights were calculated by using each household weight, multiplied by the number of children in the house, to count all children equally.

9. Standard errors for percentages were calculated according the methodology specified by the US Census Bureau: https://www2.census.gov/programs-surveys/demo/technicaldocumentation/hhp/Phase2_Source_and_Accuracy_Week_17.pdf (accessed November 25, 2020). This entailed using a weights file produced by the Census Bureau, containing 80 household weights for each respondent. Each outcome for each group was initially calculated using the main household weight to create the point estimate. Subsequently, each outcome was calculated 80 times, using each of the 80 household weights. The standard errors for each estimate was generated using the below formula, where $\hat{\theta}$ is the point estimate and $\theta_{i}$ is the estimate calculated from each alternative household weight:

$$
\operatorname{Standard} \operatorname{Error}(\hat{\theta})=\operatorname{sqrt}\left(\frac{4}{80} \sum_{i=1}^{80}\left(\theta_{i}-\widehat{\theta}\right)^{2}\right)
$$




\section{Methodological Considerations}

1. Percentages reflect US households surveyed in the August 19 - October 26 window, from waves 13 through 17 of the survey. Each wave of the survey is equally weighted. The total sample size for this period was $n=502,692$ respondents. The sample size of the subset of households with children actively participating in online learning was $n=72,179$ respondents. See all sample sizes for each subgroup in the supplemental table below.

2. Standard errors were created by the Census Bureau using successive difference replication. They primary capture the magnitude of sampling error, although some non-sampling error is additionally measured. Bias introduced by non-sampling error is not captured.

3. All questions were answered by one representative from each household. In households with multiple children, there could be nuances between children that are missed by the survey design. It is likely reasonable to assume that most children in the household have similar access to the internet and computer technology. It may also be reasonable to assume that onlinelearning decisions affect children in the household equally. However, this does represent a limitation that should be taken into consideration when interpreting study results.

4. More survey methodological details are available here: $h t t p s: / / w w w . c e n s u s . g o v / p r o g r a m s-$ surveys/household-pulse-survey/technical-documentation.html (accessed November 25, 2020).

5. Additionally, it is important to note that further research will be required to more directly measure learning gaps stemming from COVID-19 related educational disruptions. While important, access to the internet and a computer are only a proxy for learning outcomes. 


\begin{tabular}{|c|c|c|c|}
\hline Race/Ethnicity Group & Parental Education & Sample Size (\# of Children) & Estimate $(95 \% \mathrm{Cl})$ \\
\hline All & All & 72179 (140367) & $10.1 \%(9.6 \%-10.6 \%)$ \\
\hline All & Graduate & $20254(39052)$ & $3.9 \%(3.5 \%-4.3 \%)$ \\
\hline All & Bachelor's & $21713(41940)$ & $5.2 \%(4.6 \%-5.9 \%)$ \\
\hline All & Associates or Some College & $21853(42450)$ & $10.8 \%(10.0 \%-11.6 \%)$ \\
\hline All & Highschool & $6940(13774)$ & $13.1 \%(12.0 \%-14.3 \%)$ \\
\hline All & Less than Highschool & $1419(3151)$ & $20.3 \%(16.5 \%-24.2 \%)$ \\
\hline Asian, Non-Hispanic & All & $5049(9131)$ & $3.8 \%(2.8 \%-4.9 \%)$ \\
\hline Asian, Non-Hispanic & Graduate & $2123(3769)$ & $1.9 \%(1.2 \%-2.7 \%)$ \\
\hline Asian, Non-Hispanic & Bachelor's & $1803(3257)$ & $2.8 \%(2.0 \%-3.5 \%)$ \\
\hline Asian, Non-Hispanic & Associates or Some College & $822(1526)$ & $4.5 \%(2.5 \%-6.6 \%)$ \\
\hline Asian, Non-Hispanic & Highschool & $225(427)$ & $7.2 \%(1.8 \%-12.7 \%)$ \\
\hline Asian, Non-Hispanic & Less than Highschool & $76(152)$ & $10.3 \%(3.5 \%-17.0 \%)$ \\
\hline White, Non-Hispanic & All & $48421(94215)$ & $8.0 \%(7.5 \%-8.5 \%)$ \\
\hline White, Non-Hispanic & Graduate & $14461(28336)$ & $3.6 \%(3.1 \%-4.1 \%)$ \\
\hline White, Non-Hispanic & Bachelor's & $15682(30758)$ & $4.8 \%(4.1 \%-5.5 \%)$ \\
\hline White, Non-Hispanic & Associates or Some College & $13694(26236)$ & $8.9 \%(8.1 \%-9.6 \%)$ \\
\hline White, Non-Hispanic & Highschool & 4067 (7799) & $11.9 \%(10.5 \%-13.3 \%)$ \\
\hline White, Non-Hispanic & Less than Highschool & $517(1086)$ & $16.5 \%(10.8 \%-22.2 \%)$ \\
\hline Hispanic & All & 8910 (17873) & $12.4 \%(10.8 \%-13.9 \%)$ \\
\hline Hispanic & Graduate & $1461(2840)$ & $5.3 \%(3.7 \%-6.9 \%)$ \\
\hline Hispanic & Bachelor's & $1970(3703)$ & $7.5 \%(4.7 \%-10.3 \%)$ \\
\hline Hispanic & Associates or Some College & $3439(6915)$ & $13.0 \%(10.9 \%-15.2 \%)$ \\
\hline Hispanic & Highschool & $1439(3055)$ & $11.6 \%(9.2 \%-14.0 \%)$ \\
\hline Hispanic & Less than Highschool & $601(1360)$ & $17.6 \%(11.5 \%-23.7 \%)$ \\
\hline Other/Mixed, Non-Hispanic & All & $3310(6768)$ & $15.2 \%(12.5 \%-17.9 \%)$ \\
\hline Other/Mixed, Non-Hispanic & Graduate & $653(1285)$ & $7.3 \%(4.0 \%-10.7 \%)$ \\
\hline Other/Mixed, Non-Hispanic & Bachelor's & $835(1646)$ & $7.1 \%(4.0 \%-10.3 \%)$ \\
\hline Other/Mixed, Non-Hispanic & Associates or Some College & $1324(2731)$ & $15.7 \%(11.9 \%-19.5 \%)$ \\
\hline Other/Mixed, Non-Hispanic & Highschool & $431(934)$ & $19.4 \%(13.0 \%-25.9 \%)$ \\
\hline Other/Mixed, Non-Hispanic & Less than Highschool & $67(172)$ & $27.2 \%(7.8 \%-46.6 \%)$ \\
\hline Black, Non-Hispanic & All & $6489(12380)$ & $15.6 \%(13.5 \%-17.7 \%)$ \\
\hline Black, Non-Hispanic & Graduate & $1556(2822)$ & $5.9 \%(4.1 \%-7.8 \%)$ \\
\hline Black, Non-Hispanic & Bachelor's & $1423(2576)$ & $6.9 \%(4.6 \%-9.3 \%)$ \\
\hline Black, Non-Hispanic & Associates or Some College & $2574(5042)$ & $13.6 \%(11.1 \%-16.2 \%)$ \\
\hline Black, Non-Hispanic & Highschool & 778 (1559) & $18.9 \%(14.6 \%-23.1 \%)$ \\
\hline Black, Non-Hispanic & Less than Highschool & $158(381)$ & $35.5 \%(24.1 \%-47.0 \%)$ \\
\hline
\end{tabular}

Supplemental Table. Percent of children (age 0-18) learning online whose parents reported they had inadequate access to the internet or a computer, in the Fall 2020 school period (August 31 -October 26), shown by parental race and parental education gradient. All intersections are shown as well as marginal values. All percentages represent survey-weighted values. The sample size is shown, representing the number of respondents, alongside the number of children living with those respondents, shown in parentheses. The point estimate is shown along a $95 \%$ confidence interval for each quantity. 


\section{Data and Code Access}

All data used in the analysis are publicly available at: https://www.census.gov/programssurveys/household-pulse-survey/datasets.html (accessed Nov 25, 2020).

The $\mathrm{R}$ code used to run the analysis is available below (to be made available on a public GitHub page, along with the data, for final publication). Note that the root directory must be set the same location as the downloaded data, to use the code.

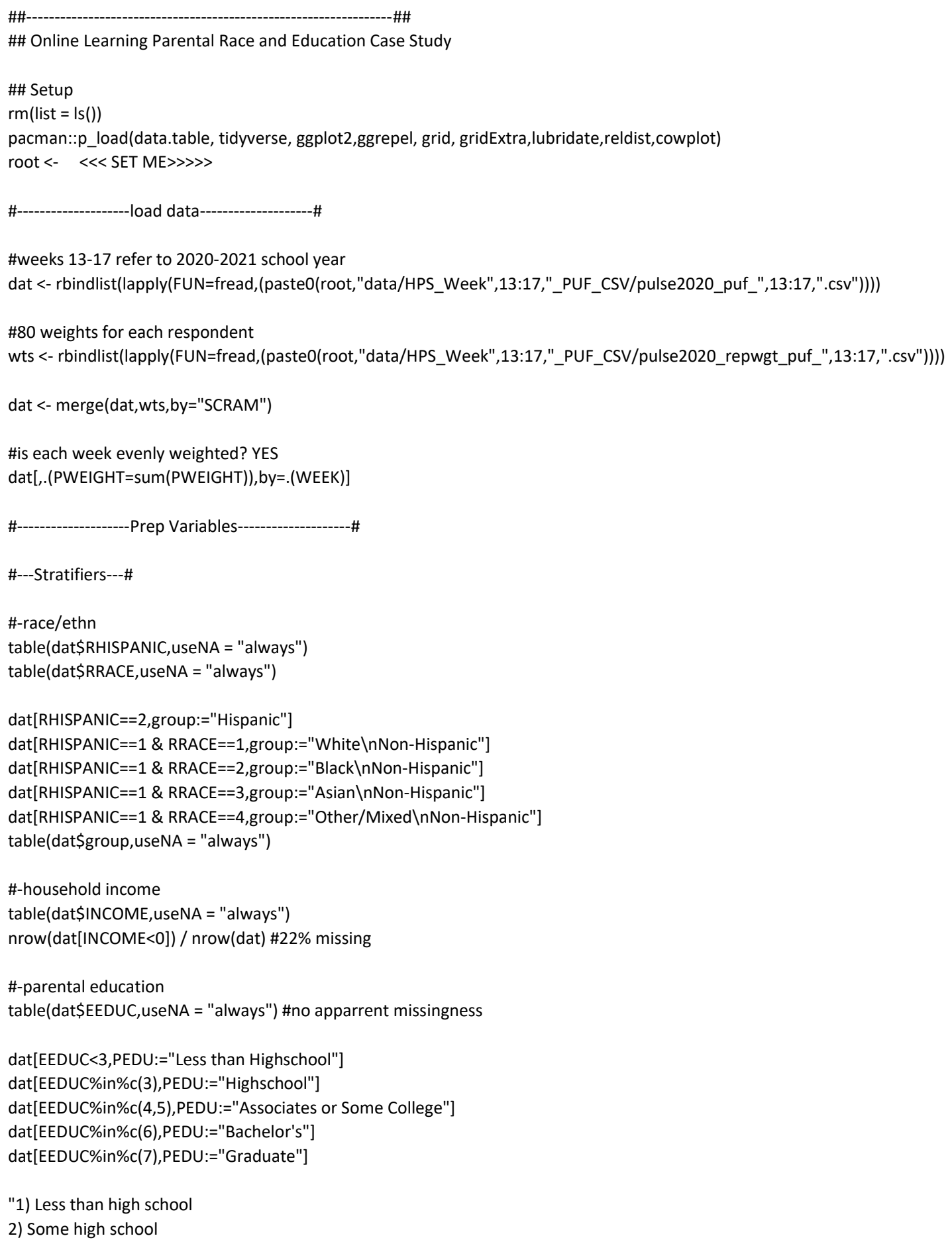


3) High school graduate or equivalent (for example GED)

4) Some college, but degree not received or is in progress

5) Associate's degree (for example AA, AS)

6) Bachelor's degree (for example BA, BS, AB)

7) Graduate degree (for example master's, professional, doctorate)"

\#number of kids

table(dat\$THHLD_NUMKID, useNA = "always") \#no apparrent missingness

dat[,numk:=THHLD_NUMKID]

\#---Outcome---\#

\#no computer or internet, among only those distance learning

dat[TEACH2==1 \& (COMPAVAIL \%in\% c $(1,2)$ \& INTRNTAVAIL \%in\% c $(1,2))$, dist_noresource_prop:=0]

$\operatorname{dat}[$ TEACH2 $==1$ \& (COMPAVAIL \%in\% c(3,4,5) | INTRNTAVAIL \%in\% c(3,4,5)), dist_noresource_prop:=1]

\#\%online learning

dat[,dist:=0]

$\operatorname{dat}[$ TEACH2 $==1$, dist: $=1]$

\#percent of kids in online school

\# online $<-$ dat[numk>0\&(ENROLL1==1 |ENROLL2==1)]

\# weighted.mean( $\mathrm{x}=$ online $\$$ dist, $\mathrm{w}=\mathrm{online} \$ \mathrm{HWEIGHT*}$ online\$numk)

\#subset to only parents with $>0$ children, who report their children are distance-learning

kid $<-\operatorname{dat}[($ numk $>0 \&(E N R O L L 1==1 \mid$ ENROLL2==1))]

\#Check Missingness

nrow(kid[is.na(dist_noresource_prop)]) / nrow(kid) * 100

\#subset to only needed variables

kid <- kid[,c("dist_noresource_prop","dist","numk","group","PEDU","HWEIGHT",pasteO("HWEIGHT",1:80))]

\#long on weights

kid <- melt.data.table(kid,id.vars=c("dist_noresource_prop","dist","numk","group","PEDU"),variable.name = "weight_n",value.name $=$ "weight")

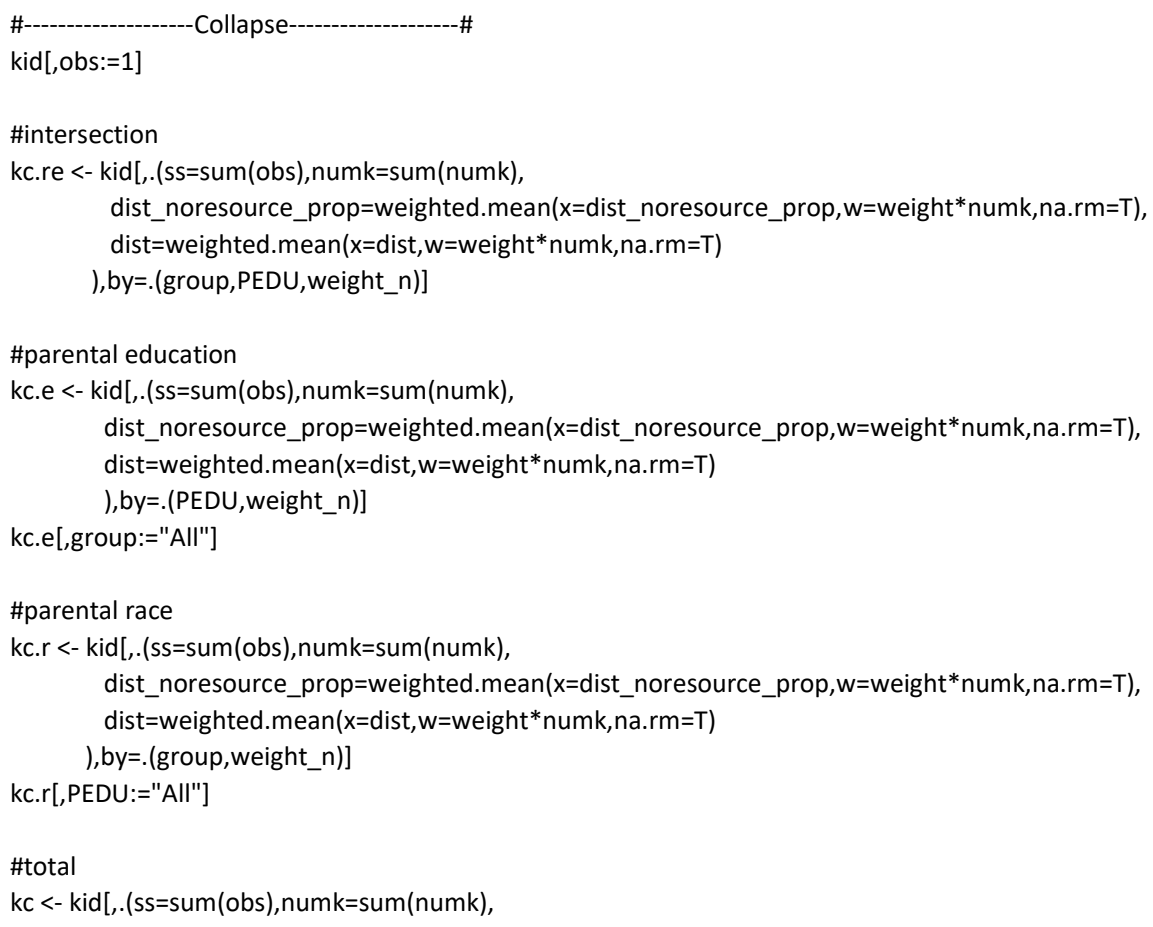




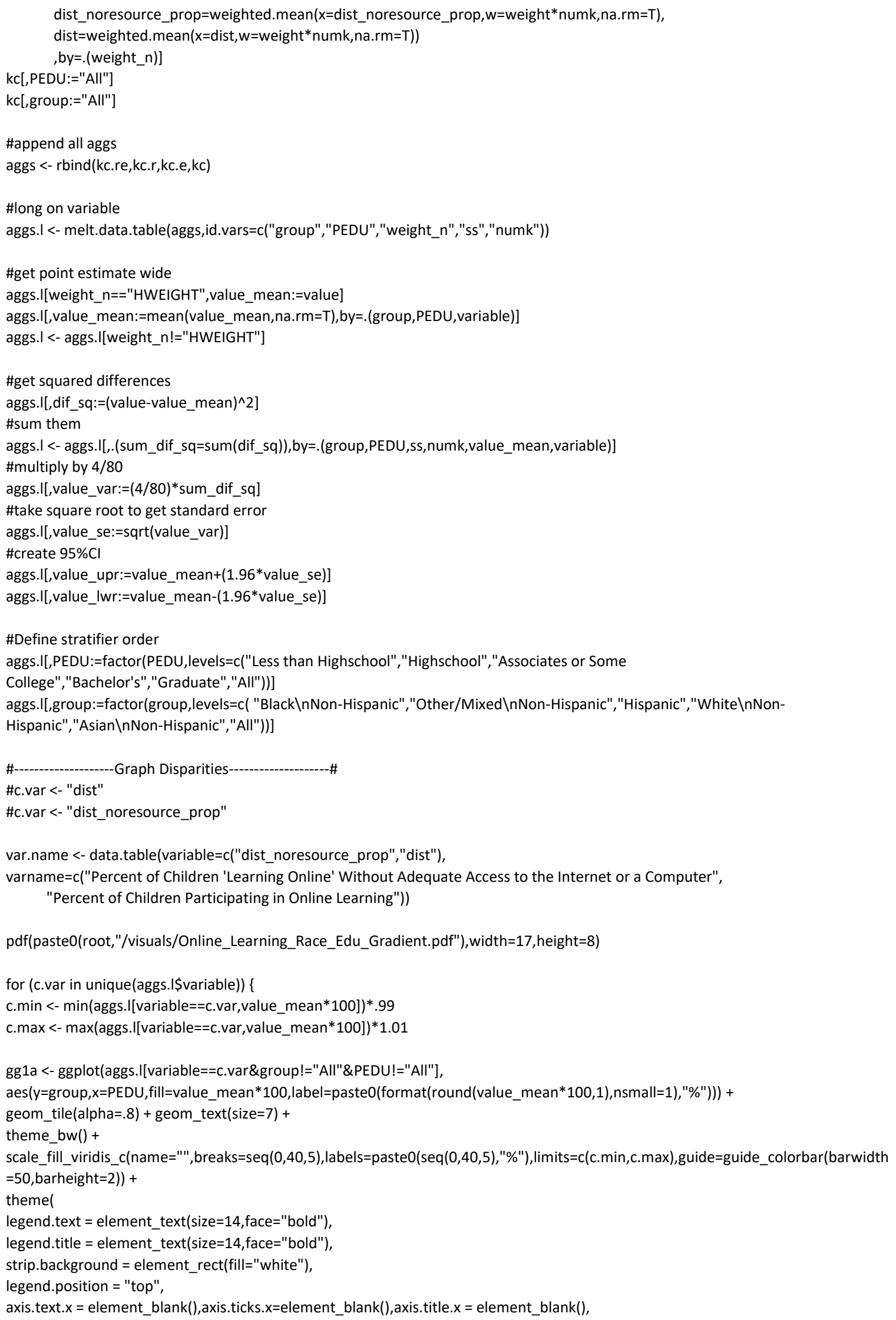




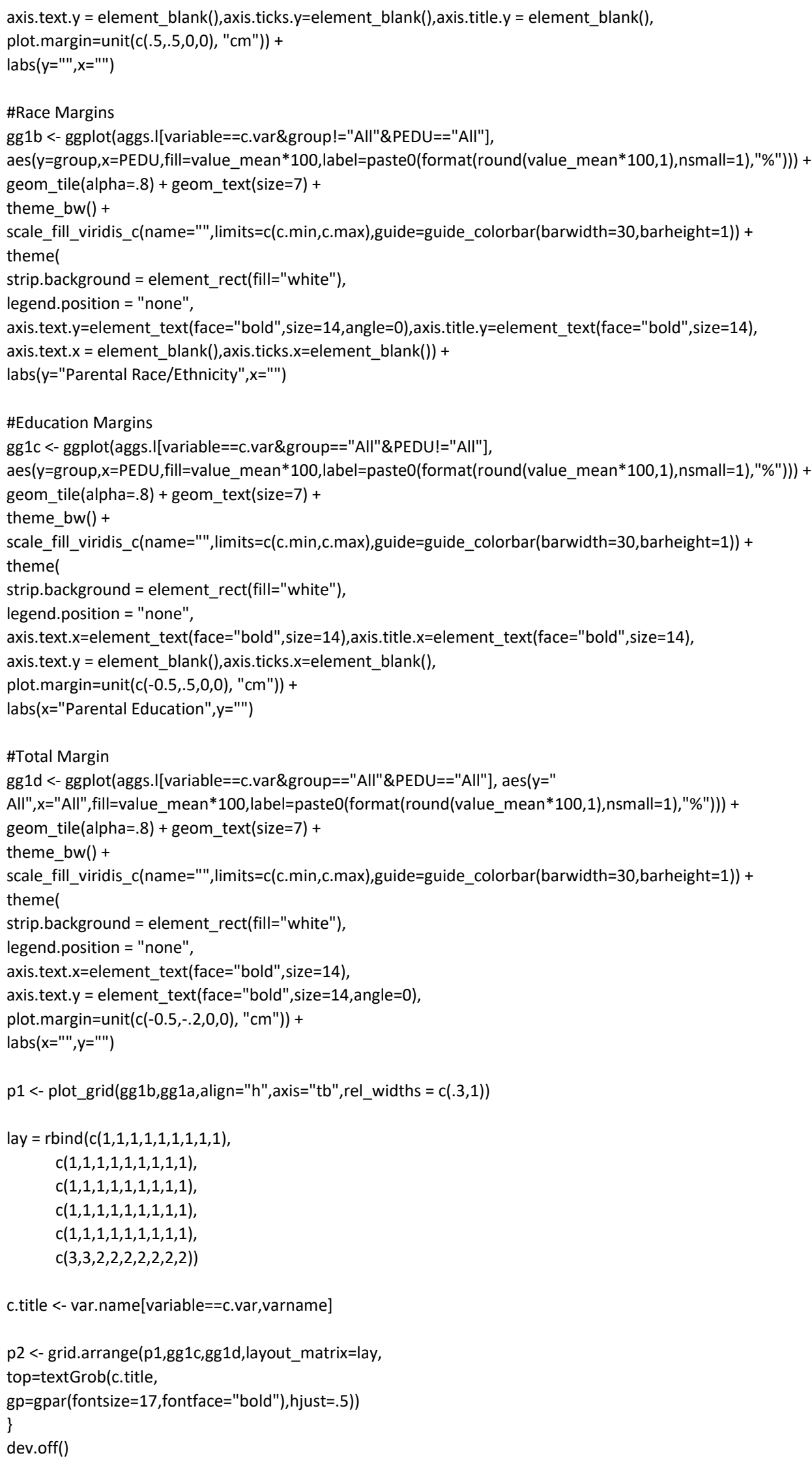


\#Supplemental Table - Sample Size in Each Group

aggs.I[,sample:=paste0(ss," (",numk,")")]

aggs.|[,estimate:=paste0(format(round(value_mean*100,1),nsmall=1),"\% (",

format(round(value_Iwr*100,1),nsmall=1),"\% - ",

format(round(value_upr*100,1),nsmall=1),"\%)")]

tbl <- aggs.I[,c("group","PEDU","variable","sample","estimate")]

tbl $<-$ tbl[order(-group,-PEDU)]

tbl[,group:=gsub(group, pattern=" \n",replacement=", ")]

$\operatorname{View}(\mathrm{tbl})$

write.csv(tbl,paste0(root,"visuals/supplemental_table.csv"), row.names=F) 